\title{
ARTÍCULOS
}

\section{MOVILIDAD, SUJETO Y PRÁCTICA SOCIAL. OBSERVACIONES EN UNA ESTACIÓN DE AUTOBUSES.}

\author{
MOBILITY, SUBJECT AND SOCIAL PRACTICE. OBSERVATIONS \\ IN A BUS STATION.
}

\author{
José Luis Anta Félez ${ }^{1}$
}

Universidad de Jaén. Área de Antropología Social

Recibido: 27 de agosto de 2019; Aprobado: 27 de agosto de 2020

Cómo citar este artículo / Citation: Anta Félez, José Luis. 2021. «Movilidad, sujeto y práctica social. Observaciones en una estación de autobuses». Disparidades. Revista de Antropología 76(1): e011. doi: <https://doi.org/10.3989/dra.2021.011>.

RESUMEN: A través de la estación de autobuses de la ciudad de Jaén, se desgrana la genealogía reciente de esta ciudad: su territorio, su conformación histórica y aparataje simbólico. Como parte de los edificios creados por el desarrollismo franquista, con los cambios recientes hacía la idea de patrimonio histórico, se realiza un acercamiento, también, a su dinámica interna, como un teatro de constantes interacciones simbólicas. Por último, se centra todo el trabajo en entender, a través de este espacio estratégico y privilegiado para la micro investigación social, el concepto de movilidad, la idea de transporte, de interconectividad y todas las posibles gestiones físicas y subjetividades con las que trabaja el Estado, la empresas poscapitalistas y sus formas híbridas, y que tienen como resultado un constante orden normativo, disciplinamiento, vigilancia, mercantilización, control del riesgo y transtradicionalidad, tanto sobre los cuerpos de los sujetos, como en las prácticas que generan.

PALABRAS CLAVE: Estación de autobuses; Jaén; Etnografía; Movilidad; Urbano; Disciplina; Riesgo; Sujeto

ABSTRACT: Through the bus station of the city of Jaen, the recent genealogy of this city is broken down: its territory, its historical conformation and symbolic apparatus. As part of the buildings created by Franco's development, with recent changes towards the idea of historical heritage, an approach is also made to its internal dynamics, such as a theatre of constant symbolic interactions. Finally, all work is focused on understanding, through this strategic and privileged space for social micro research, the concept of mobility, the idea of transport, interconnectivity and all possible physical and subjectivities with which the State, post-capitalist companies and their hybrid forms, and that result in a constant normative order, discipline, vigilance, commercialization, risk control and trans-traditionality, both on the bodies of the subjects, as in the practices they generate.

KEY WORDS: Bus Station; Jaen; Ethnography; Mobility; Urban; Discipline; Risk; Subject

Copyright: (C) 2021 CSIC. Este es un artículo de acceso abierto distribuido bajo los términos de la licencia de uso y distribución Creative Commons Reconocimiento 4.0 Internacional (CC BY 4.0).

1 Correo electrónico: jlanta@ujaen.es. ORCID iD: <https://orcid.org/0000-0001-7063-5288>. 
Down to the bus station

I looked upon the wall

My money was to light people

Couldn't go nowhere at all

See I'm a bluesman

But a good man, understand.

(B. B. King. Blues Man)

\section{MOVILIDAD Y ESPACIO PRIVILEGIADO}

Para una sociedad como la nuestra, que ha hecho del movimiento una de sus formas de identidad, y que además establece en el tránsito permanente una de sus grandes apelaciones, tiene que existir zonas privilegiadas donde toda su ideología se visualiza de una manera total. Zonas que por su especial relevancia hagan, construyan y subjetivizan los conceptos sociales como si de una máquina de captura se tratara, donde el etnógrafo no puede hacer otra cosa que gozar de su trabajo: observar esas realidades como una metáfora textual de una cultura precisa, a la vez que desentraña unos determinados valores y conceptos e ideas significativos y representativos de aquello que tiene delante. Esos lugares que se preñan de tropos culturales, donde lo que representan está en relación con lo que muestran, estructuras sociales que relacionan los conceptos y su posible significado. Si en tiempos pretéritos la catedral, más acá la fábrica y casi ayer la cárcel o el hospital fueron ese lugar, esa zona fuerte de lo social, hoy no podemos dejar de pensar en cómo nos representamos acaso en el supermercado o en poscapitalista y donde el concepto de lo contemporáneo tiene un sentido total y se muestra, digamos, sin tapujos (Urry 2007). De esta manera, entendemos que el concepto de seguridad, movilidad, representación, modernidad, mercado o disciplina se encuentran alojados de manera representativa, normativa y práctica en esos espacios, un tanto negados, asépticos y sobrevigilados, y que en el aeropuerto llega a su máxima exageración y radicalidad (Güller y Güller 2002; Potthast 2012). En efecto, es ahí donde lo que esta sociedad dice ser toma carta de presentación, forma concreta y material. El aeropuerto parece, en este sentido, la institución metafórica clave del mundo contemporáneo poscapitalista.

Sin embargo, podríamos decir que hay una serie de espacios que mantienen una lógica paralela a lo planteado por el aeropuerto y que, sin embargo, son restos de un pasado, incluso de un espacio-presente, que representan múltiples conceptos ambivalentes. La estación de autobuses de Jaén, una pequeña ciudad de la Andalucía interior (el Sur en su sentido más amplio), es uno de esos lugares que sirve de representación de un mundo que muestra su cara más decadente, clasista y furibunda. Pero que, a su vez, como en el aeropuerto, se asienta el lugar central del discurso de la modernidad. La estación de autobuses trasciende su propia idea de ser un espacio funcional, aunque tenga el empeño de apuntalar ese discurso, para mostrar una serie de elementos que son centrales en la sociedad que lo circunda, usa e, incluso habita: desde el concepto de movimiento al de tránsito, desde la idea de ordenamiento social a la de emigración. Todo lo social parece concentrarse en este lugar, como práctica y representación. Consecuentemente, podemos decir que es un espacio clave para la mirada no sólo de la teoría social, sino ante todo de la propia sociedad que lo gestiona y agencia.

Tenemos entre manos, por lo tanto, dos cosas: por un lado, un lugar privilegiado de lo social, con lo que supone para ver y para poner a prueba nuestros conceptos. Y por otro lado, un espacio donde ocurren muchas cosas y que, en última instancia, es una muestra muy coherente de cómo funciona y se articula el mundo contemporáneo poscapitalista, ciertamente en un lugar tan exótico como es la ciudad de Jaén. En este sentido, la propia arquitectura que lo conforma como un proyecto funcional y simbólico determinado, así como el espacio urbano circundante, son parte a su vez de un criterio histórico, genealógico y etnográfico, que tiene su propio contenido, y donde se ha puesto a prueba como un discurso funcional que lo explica: el de servir de lugar donde funciona el principio y fin de las líneas de autobús que conectan Jaén capital con el resto de las poblaciones y fuera de esta, con una gran cantidad de otros lugares. Pero también hay una historia social que da cuenta de qué lugar ocupa Jaén en una red más extensa de espacios regionales, provinciales y nacionales, así como el decaimiento de lo público, visible sin reparos en la constante desatención por el transporte basado en el sistema ferroviario (Seijo 2006).

La estación de autobuses es también un lugar para observar las causas de las políticas municipales, regionales y nacionales. No menos para prestar atención a cómo se aborda el urbanismo e incluso, cuales son los intereses inmobiliarios de un espacio en constante remodelación y cambio. La estación 
de autobuses se mueve en múltiples planos, cristalizando desde una mirada muy pequeña, que da cuenta de esa micro-sociología del fascinante comportamiento humano, hasta los niveles simbólicos que se concentran en torno a la idea de mercado, el movimiento y el desarrollo, pasando por las posibilidades de hacer una historia de corte social a través del espacio y su edificio, y cómo se ha transformado y terminando con una mirada de gran espectro sobre los grandes planes de carácter político que no parece posible en un mundo en red, interconectado y balanceado en ideas que tienen que ver con el centro-periferia, la dependencia y la gestión de lo público (Daniels y Warnes 1983; Fernández Durán 1980). Así, la estación de autobuses concentra ese discurso del Estado de Bienestar, junto con la escuela, la policía y el hospital, que se entienden como derechos, junto a otros que han de garantizarse desde las políticas públicas: la educación, la seguridad, la sanidad, los servicios sociales y el transporte. Podría decirse, que sí el aeropuerto es el espacio privilegiado y central de la sociedad poscapitalista, la estación de autobuses lo es de la sociedad que concentra su discurso en el Estado de Bienestar. Pero a su vez hay que recordar que la decadencia de lo público es patente, tal cual en sus productos y sus valores, la amenaza de la privatización y mercantilización de este espacio que no es sino el de la propia historia de los objetos públicos-privativos; una vez más, evidenciamos el derribo de la idea de bien común, espacio conceptual donde se enclava todo esto que aquí estudiamos.

Pero esta decadencia no es sólo el producto de unas ideas en retirada, sino que es visible también en el mantenimiento de la parte arquitectónica y sobre todo, en mucho de lo que en su interior ocurre, con un funcionamiento basado en un mercado basado en ideas keynesianas, digamos, de primer orden, con un sistema laboral y de comercialización que es una auténtica reliquia de los tiempos en que el capitalismo de corte estatista era un gestionador de bienes, servicios, recursos humanos y materiales (Herce y Miró 2002). Obviamente, un Estado que no dudó en ofrecer ayudas y coartadas narrativas a las sociedades mercantiles que le eran afines a sus intereses. También un espacio, este de la estación, donde ocurren muchas cosas, unas en tránsito y otras fijadas a ideas e interpretaciones de la modernidad. Un espacio para los sentimientos y la política. Un espacio que no puede ser otra cosa que una atalaya desde la cual observar de manera privilegiada lo social, lo político y lo económico en un mundo extraño, exótico y en constante transformación. En última instancia todo parece remitir a una cierta idea de orden del territorio, de materialidad desde la que se asienta las redes, la identidad y las miradas a la sociedad como espacio para la vida (Zárate 1997). Por otro lado, el debate sobre el modelo de ciudad que se plantea en un nivel técnico, cuanto más político, tiene en el transporte uno de sus ejes de discusión (Crespo y Moya 2012; Seguí 2004; Martínez 2004), tanto en moverse como la manera de hacerlo. La movilidad es ese gran concepto contemporáneo sobre el que ha funcionado la idea de que hay que controlar la sociedad, a la vez que permitirle una cierta expansión (Urry 1999). Convertida así, en un sentimiento gestionado y donde la movilidad contemporánea bien podría ser entendida como la ausencia de una experiencia de "recorrer" y sólo un deseo de "Ilegar". En consecuencia, la movilidad no parece tener un sentido como experiencia y subjetividad del viaje, reduciéndose a un mero ejercicio de transporte. De ahí su importancia en cuanto a los objetos que usa y dispone, el autobús tanto el urbano como el interurbano, lejos de ser un anacronismo es una idea fuerte de las posibilidades de desplazarse. Por lo tanto, la estación de autobuses, a lo que hay que sumar la motorización de todo el espacio urbano, es un lugar determinante de toda esta ideología del movimiento contemporáneo (Horta 2019; Jacobs 2011: 377-410).

Claro que también está la idea de transporte, de la necesidad de tener instrumentos eficaces, democráticos y asequibles para moverse. Todo un debate que sin duda hay que tener en cuenta. Sin duda, porque el pasado está estructurado en la hegemonía del monopolio capitalista. En los últimos veinte años todo un núcleo de geógrafos, urbanistas y expertos varios ha irrumpido dejando claro que, primero, el transporte es parte del Estado de Bienestar y segundo, que la movilidad es un bien que conforma las ciudades. El concepto de "derecho a la movilidad" tiene sus orígenes en la sociología urbana de Lefebvre (1976: 127-144), quién señaló la importancia de la movilidad como tema social y político, en la medida que remarca las condiciones del acceso, o no, a una economía más distributiva, remarcando la democratización de los espacios a los que se le vincula, y estableciendo redes de necesidades y servicios con respecto a los espacios de 
la vida social, laboral, de ocio y de vivienda. Es evidente que la red de transporte, en tanto representación de las ideas conceptuales de la movilidad en el espacio presente, se puede ver como un recurso que establece una serie de diferenciaciones con respecto a la equidad, la justicia y el acceso a la riqueza. Una suerte de entramado de saberes y demostraciones que tienen que ver con una cierta geografía de los derechos democráticos (Gutiérrez 2010; Herce 2009 y 2010). En este sentido, la movilidad es por una parte, precondición de los otros derechos, y por otra, una especie de derecho genérico con una importancia social, política y económica creciente. No se puede olvidar que es, en cierta medida, uno de los factores de la vida cotidiana en un mundo altamente globalizado, líquido y movedizo. A la par que el debate del transporte entra de lleno en los condicionantes económicos más globales, como el uso de combustibles, el impacto medioambiental o los sistemas de seguridad de las personas (Natal 2003). Todo un mundo de retos para la sociedad actual y que tiene una importancia grande en la manera en cómo se piensan los ciudadanos en relación a sus recursos y a su contenido social y cultural.

\section{LA CONSTRUCCIÓN DE UN ESPACIO ETNOGRÁFICO}

Aquí tomo la estación de autobuses de la ciudad de Jaén como uno de esos espacios privilegiados para la mirada de la sociedad poscapitalista contemporánea. Consecuentemente es tratado como absoluto etnológico: un lugar que no sólo es una representación de lo social, sino también donde la sociedad en que se enmarca lo utiliza como una gran pantalla de sus ideas, valores y prácticas, concentrando, al final, lo social en sí mismo. Una zona cultural que también produce y reproduce multitud de geografías sentimentales, un constante derroche de despedidas y bienvenidas, de inicios y rupturas, de relaciones que terminan y acaban. Yo mismo soy un ejemplo de ello. Esta estación fue el primer sitio que pisé de Jaén, hace ya más de dos décadas, cuando llegué para encargarme de una plaza de profesor de antropología social en su universidad. Pero, como usuario del transporte público, también es lo último que piso cuando salgo de Jaén. Y nadie puede negar que en esta decadente y simbólica estación se dan miradas cruzadas de múltiples elementos que lo hacen, a los ojos del etnógrafo, un espacio privilegiado. De alguna manera no quería caer en la seductora, pero realmente falsa, idea de que estamos ante un no-lugar, ese entramado conceptual de Augé (1993). Esta estación de autobuses no es sólo un lugar de paso, acaso un espacio donde no puede ocurrir nada porque no hay sitio para el pensamiento; pero también tiene que ser visto como un referente de y para la ciudad, en la medida que mecaniza una de las maneras claves de entrar y salir, además, se trata de un lugar referencial de la historia, las prácticas y las formas de entender las actividades de los sujetos de esta parte del mundo.

A sus virtudes como espacio social se une que tiene, de alguna manera, una cierta comodidad y amabilidad para el estudio. En mi caso no sólo cumplo con la idea de hacer participación -soy un usuario frecuente de la estación de autobuses-, sino que permite una más que cómoda observación (la cual sistematicé en el transcurso de un año y medio entre 2016 y 2018), dado que los espacios interiores y exteriores están bien definidos, los comportamientos están muy normativizados, las funciones muy regularizadas y los actores son claramente visibles. Pero esta facilidad y transparencia no sería más que una pura amabilidad si no fuera porque la propia estación es una constante histórica, que desde su construcción en los años 40 del siglo XX ha sido permanentemente utilizada. Se une así a las posibilidades de ver lo minúsculo de la obra teatral que allí se representa a diario con un marco que prácticamente no ha cambiado a lo largo de la historia reciente de Jaén. Esta doble dimensión lo convierte, en efecto, en un espacio muy concreto para ver la realidad, los valores, los comportamientos y por supuesto, la aparición y los cambios contextuales de los conceptos con los que se vive y construye la modernidad.

Pero obviamente no hablamos de entender la estación de autobuses como un laboratorio social, ni mucho menos, no se trata de poner a prueba nuestra hipótesis, en el caso de partir de ahí, sino más bien lo contrario: tomar la estación en un doble sentido, por un lado, como una expresión y respuesta de la sociedad local a problemas globales y sobre todo, de ver la estación como un espacio puramente social, donde lo que ocurre tiene que ver con las ideas, símbolos y prácticas de los sujetos que la usan, así como los conceptos propios que definen el mundo contemporáneo local. En este sentido no trato tanto de recrear una historia (etnológico-estructural), sino de ver el presente, primero, como el resultado de un 
proceso histórico, dinámico y complejo y segundo, de articular la lógica de esos cuerpos sujetos a las subjetividades, a las dinámicas de la movilidad, a las políticas públicas y privadas del mercado y a las formas de vivir en sistemas sociales segmentados por factores de edad, género, etnia y/o clase social. En última instancia, se trata también de dar cuenta a través de las descripciones etnográficas y sus interpretaciones, de la ausencia de una experiencia personal y colectiva en las actividades cotidianas que nos definen como sociedad (Anta 2013).

El método etnográfico, sumándose a esa lógica de la que hablan Velasco y Díaz de Rada (2009), no puede ser sólo un proceso de descripción, sino ante todo un espacio académico e interpretativo donde podemos observar también los procesos de transformación y dar cuenta de la verdad de manera crítica. El trabajo de campo no es sólo un mirar "legitimado" sino, seguramente, el compromiso con la búsqueda de las formas lógicas que gobiernan los mundos locales. La etnografía, como la que yo defiendo aquí, no es una actividad ritual dentro de un laboratorio étnico, sino el punto desde el que arrancan las formas de pensar críticamente el presente en el que vivimos (Taplin, Scheld y Low 2002). No se trata de ver gentes que hacen, sino de ver sujetos que practican, y consecuentemente de hacer conjeturas conceptuales que sean válidas en la medida que denotan cuál, dónde, quién y cómo se interpreta este presente. Hay que describir, traducir, explicar e interpretar, pero también tenemos que crear una voluntad de teorizar y criticar el marco instituido que tomamos como "objeto" privilegiado de nuestra mirada y no olvidar que lo habitan sujetos que, como nosotros, tienen sobre todo la capacidad de sufrir.

Cuando se vive el mundo contemporáneo europeo, aunque sea desde el profundo Sur, es obvio que la mirada sólo puede darse en la medida que lo hacemos sobre objetos e ideas que se mueven o de sujetos que se desplazan. No es sólo la movilidad como concepto, el cual es sin duda clave, sino también en función de qué se hace y la manera en cómo se produce. De hecho, vemos el mundo moverse desde lugares en movimiento. El tren, el autobús, el automóvil no sólo son claves para la mirada, sino que permiten crear el lugar preciso desde el que mirar. Y esta mirada, desde el autobús, ya no es una experiencia, ya no podemos decir que describe algo con una cierta capacidad de transformar la realidad, en última instancia al dejar claro que nos transportarnos como medio de vida, todo lo que hacemos desde la observación no tiene ningún valor. Muy por el contrario, la mirada desde el autobús (en la medida que es también una metáfora de este artículo) es sobre todo una síntesis de lo qué es el mundo y la manera en cómo este está diseñado fuera del sujeto. Desde un autobús todo lo de fuera es sólo un dispositivo de diseño: un paisaje sintetizado de la subjetividad contemporánea. Curiosamente Luis D'Aubeterre (2012) en su estudio hermenéutico de la forma de ser de los venezolanos lo hace en y desde una línea de autobuses de media distancia (pullman) y observa, no sin un cierto grado de ironía, que hay dos ritmos diferentes en la estación de autobuses de Caracas: por un lado, un cierto grado desorden en torno a lo que ocurre en el espacio localizado, donde los vendedores, la música, incluso el ruido, y los pequeños pícaros dan color y alegría al espacio y donde las relaciones sociales tienden a ser complejas y llenas de significados locales. Y por otro, el orden casi matemático y claramente "europeo" del interior de los autobuses, donde los sujetos tienden a mantenerse en una zona gris de bajo impacto e interacción social. Un contraste, dentro-fuera, que es en definitiva una metáfora que preconiza la vida en el movimiento de los sujetos en la sociedad poscapitalista.

\section{LA ESTACIÓN COMO UNA EXPERIENCIA}

La experiencia de lo urbano tiene que ser atacada consiguientemente, en la propia idea de que todo empieza y acaba en una estación de tren, de autobús, en un aparcamiento, en la parada del transporte (Chambers 1986: 18-19), que nos lleva de aquí para allá y nos permite el sabernos del lugar o de fuera. Una estación de autobuses sólo puede ser el principio y el fin de todo lo que tenga que ver con una narrativa. Es el lugar donde el sujeto entra en la idea del tránsito, en la falta de corporalidad que le ha de llevar de aquí para allá, por eso la estación de autobuses es clave para entender lo social, porque transforma a los sujetos en narrativas corporales, en viajeros. Se puede decir que es una suerte de maquinaria que tiene la capacidad de transformar el objeto cuerpo. La estación cumple, en este sentido, con un elemento añadido muy interesante, es un lugar donde todo parece meticulosamente repetitivo, previsible y ajustado a un horario. Y sin embargo, es más un lugar de construcciones míticas que de rituales, en cierta medida porque el ritual responde 
aún espacio de interacción social en función de los intereses de este mercado, mientras que los sujetos se ven reconstruidos en una categoría de movilidad, viajeros y cuerpos míticos. Todo concuerda porque la estación fuera de cualquier otra función es un espacio donde se cumple con la idea de que la gente interactúa en función de un acontecimiento que se basa en un mito del capitalismo: todo ocurre en un lugar y tiempo prefijado.

A diferencia de otros sistemas, la estación de autobuses suple el anterior régimen donde los autobuses no tenían ni un horario claro, ni un espacio definido para parar, todo era una aproximación a lo que mil elementos ajenos determinaban, la cantidad de paradas que se hacían o el estado de las carreteras. Pero al construirse las estaciones todo quedaba establecido dentro de la vocación taylorista de producción espacio-temporal. El espacio se hacía uno y los tiempos previamente ajustados se formalizaban en forma de horarios, sin atender a ningún condicionante externo ni elementos indeterminados. El mito está creado. Es lo que Dalmaso y Coutinho (2010) entienden como un espacio productivo: en el transporte colectivo de viajeros cuando estos se bajan y se suben generan una cadena de construcciones económicas y políticas que se conforman en forma de mito: hay un lugar y un tiempo en que el viajero se montará en el autobús y otro para bajar. La vida en sociedad tiene un supuesto orden, que hace que lo urbano, en este caso, no sea más que un teatro en que los cuerpos cumplen papeles dramatizados donde desarrollan deseos e inquietudes (Goffman 1991: 191-196). De hecho, si podemos leer el texto social y la lógica civilizatoria en la estación de autobuses es porque su ritual está inicialmente creado en sus mitos, y donde todo es reducido a una narrativa de objetos y cuerpos móviles en una cierta transformación.

Claro, que a diferencia del aeropuerto, con su fascinación por lo aséptico, la paranoia por la seguridad y el plegamiento a la realidad disciplinar y de control, en la estación de autobuses los cuerpos son transformados en un bajo impacto, son cuerpos viajeros que no se desarticulan en un permanente control sino en la necesidad de entrar, salir, llegar e irse. La estación de autobuses es, en este sentido, un paso previo, pero por eso mismo, diferente del aeropuerto. Aquí los cuerpos tienen aún la idea de que son cuerpos en tránsito más que cuerpos en transformación, aquí son aún más duros, más físicos y tienen aún más capacidad de mostrarse con una apariencia de ciudadanía. En efecto, la estación de autobuses es también un transformador bio-jurídico, que permite mostrar el momento en que los cuerpos pasan del régimen de lo público, la calle, a lo privado, el autobús. Lo que significa que tras los controles de acceso hay un reconfigurador de las situaciones sociales. De hecho, cuando se compra un billete de autobús se asume la idea de que se puede pasar, se tiene derecho a un viaje, cuando en realidad lo que uno hace es asumir su lugar en la lógica del mercado poscapitalista, donde los sujetos son reducidos a un cuerpo que se transporta, a una razón jurídica basada en cuerpos-objeto que se llevan de aquí para allá.

A la vez que se da esta física de los cuerpos en transformación jurídica, concurre una bien diferente, la química de las conexiones. Cuerpos conectados y desconectados, sujetos que se dicen adiós y se besan, y cuerpos que muestran en la línea de la cola de la espera, dispuestos al contacto, generalmente breve e imperceptible. Pero una estación de autobuses es también el símbolo de una modernidad, de una voluntad de progreso basada en la conexión entre puntos, es un nodo de una sociedad que se piensa en red. De ahí que la idea de conexión, en última instancia una forma de planificación tecno-política, termine por empapar también a la idea, más decimonónica, de movilidad (Castilho 2012; Giucci y Errázuriz 2018); de movilidad permanente. La estación de autobuses, como nudo de una red, el terminal de la información, el interfaz de un complejo sistema que opera en su doble sentido, como deseo, un deseo viajero y, como metáfora de la idea de transformación de los cuerpos viajeros. Pero es la propia idea de estación de autobuses la que deja claro su voluntad de tratar a los cuerpos no por lo que son, determinando su posición, sino por lo que están haciendo, por su estado. La estación, consecuentemente, reduce el cuerpo a un nivel de hacer, un mandato en la máquina del deseo de irse, de llegar, de no quedarse.

De hecho, en la estación se puede esperar, un estado inestable y ralentizado de la materia en movimiento, que supone una tensión del sistema cuerpo, pero no se puede quedar, que se entiende como un sistema apagado y estacionario. En la estación los cuerpos no ocupan un lugar, sino que transitan a diferentes velocidades el espacio de transformación. Por eso mismo quizás no sea un lugar, sino sólo un espacio, en cierta medida, porque sus coordenadas físicas son únicamente visibles en relación al doble juego 
de lo que ocurre, de lo que sólo puede ocurrir, que es subir y bajar de un autobús, esperar o comprar un billete, acaso otras actividades marginales como la de hacer etnografía (también algo factual y transitorio). Y a su vez también, porque la estación de autobuses aprovecha esas coordenadas para hacerse presente, por medio de volúmenes arquitectónicos, en la ciudad. Espacios para los viajeros, que no tanto un lugar, que sólo es tal en la superposición del mapa sobre el territorio urbano.

\section{LA ESTACIÓN DE AUTOBUSES EN SU DINÁMICA HISTÓRICA}

La estación de autobuses es una novedad de bien entrado el siglo XX, asociada por un lado al desarrollo de las modernas ciudades y al planteamiento de la movilidad urbana e interurbana como apuesta política y por otro, como un proceso empresarial privado decidido a plantar cara al transporte nacionalizado que representaba el tren (Frax y Madrazo 2001; Millán 2001 y 2006; Montes 1999). En este sentido la estación de autobuses es en cierta medida una suerte de utopía del mundo empresarial, asociado al transporte, a la ingeniería y a las tecnologías sociales. Las estaciones de autobuses son centros legalmente reconocidos para la salida y llegada de autobuses de carácter público, regular y que transportan viajeros por carretera. Las estaciones de autobuses, además, son un complejo entramado de empresas públicas, con diferentes niveles de responsabilidad, aunque generalmente recae sobre los ayuntamientos, en su relación con otras de carácter privado, tanto en lo tocante a la explotación de la propia estación, como la de las líneas de autobuses que utilizan sus servicios. Las condiciones de comodidad y seguridad que estos centros ofrecen al viajero para la espera, facilidad en las correspondencias, servicios complementarios (bar, restaurante, locales comerciales o servicios higiénicos) e información, contribuyen a mejorar la calidad y prestaciones y obviamente, generan demandas inducidas. En cierta medida, como ocurre con prácticamente cualquier servicio prestado desde el poscapitalismo, la horizontalidad del negocio, sea cual sea, es enorme. $Y$ por eso mismo, toda empresa genera negocio a empresas paralelas $y$ consecuentemente, el entramado empresarial prácticamente no deja huecos.

Así pues, la estación de autobuses es a su vez, espacio de negocios horizontales, donde todo se convierte en un espacio privativo del mercado. Esperar al autobús se hace mirando una pantalla que informa sobre los horarios y los andenes de salida, que tiene anuncios de empresas locales, tomando un café, comiendo o gastando unas monedas en una pequeña tienda de dulces "infantiles". Así, la estación con el paso del tiempo ha perdido mucho de su sentido comercial más clásico, existía hasta hace no mucho una tienda de regalos, una de comestibles-panadería, un estanco y venta de prensa, y una cafetería restaurante... además del hotel, restaurante, cafetería-bar ubicados sobre la estación, que se ha suprimido en virtud de un tipo de negocio más global: de máquinas expendedoras y negocios fuera del espacio de la estación. No en vano la estación nunca se ha dejado de ver como un atractor económico, lo que a la corta significa negocio a su alrededor y a su costa. En este sentido para los usuarios de los autobuses la estación no es tanto un espacio funcional, cuanto más un espacio tecnológico de las economías capitalistas. Esperar el autobús no puede ser simplemente un esperar, sino una parte del propio espacio privativo del mercado, que hace del hecho esperar, también, una actividad comercial y lucrativa.

Esta idea de que estamos ante un espacio tradicional, a la vez que antiguo y obsoleto, no es tan visible en su arquitectura, cuánto más en los muchos detalles que hablan de elementos de otros tiempos, las puertas, ventanas y ventanillas de venta de billetes son de madera, la falta de un equipamiento real en tiendas y cafetería, y con la pérdida del hotel que le acompañaba, incluso con la remodelación de la plaza de acceso a un suerte de espacio vacío, no funcional, ni recreativo, todo tiene un toque que va de lo tradicional, a lo ajado, a lo sobre-usado. Y la estación parece un edificio viejo y mal diseñado, lo que responde obviamente a la falta de una previsión urbanística, cuanto más a los intereses particulares de aquellos que ven un espacio en el centro de Jaén que debería usarse para tener más comercios y menos servicios. Pero aún así la estación sobrevive con su constante idea de ser un elemento heredado de otros tiempos, y donde se combina los modernísimos medios de información por televisión de plasma puestas en las paredes con los anuncios de ópticas y compañías de seguros pintados a mano en los muros, tal cual se hacía desde los años 50.

En efecto, la estación de autobuses, como cualquiera de los espacios privilegiados por el capitalismo, no es simplemente un espacio funcional, donde de manera 
sencilla y eficaz se puede hacer uso de un transporte. No sólo es una cuestión de espacios donde se cumple con un servicio, tal cual parece rezar en su rótulo (Olalla 1977), sino que ante todo son espacios complejos donde se mueven múltiples niveles de uso y disfrute, de mercado, y de gasto, donde todo tiene múltiples lecturas, a la vez y nada es únicamente lo que parece. De hecho podemos decir que la estación de autobuses de Jaén es cuando menos, además de eso mismo, una estación de autobuses, otras cuatro funcionalidades diferentes: un espacio de negocio global, una apuesta urbanística cambiante e históricamente re-situada, la metáfora de un sociedad en constante movilidad y la representación de las políticas por un modelo basado en el transporte desde lo puramente comercial y privado. De hecho, estas cuatro realidades se concentran en todo lo que ha significado, desde los años del desarrollismo franquista, la apuesta por la movilidad planificada, centrada en el negocio privado, la individualización de las conductas y responsabilidades social, la creencia de que el Estado y sus organismos de gobierno, que sólo gestionan los sentimientos de unos ciudadanos consumidores que se las tienen que ver con los particulares intereses de las empresas, cada vez más globales, que gestionan los deseos y necesidades de los sujetos.

\section{EL CENTRO DE UN RELATO}

La historia reciente de la ciudad de Jaén es, seguramente, el texto inscrito en la genealogía propia de su estación de autobuses: nacida de un proyecto desarrollista de finales de los años 50 del siglo pasado, parece dar una apariencia de centralidad a la ciudad, pero no menos al territorio provincial. Si a esto sumamos la imposición de una serie de medidas que llevaron en los años 60 a una apuesta por el transporte por carretera siendo una parte de la idea vertebradora del territorio nacional. De la misma manera, el Ministerio de Fomento en estos años llega a un cierto trato con las empresas privadas de transporte de viajeros, las cuales nacieron en general durante las tres primeras décadas del siglo XX y que habían conformado el núcleo duro de un sistema que se decidía por un sistema mixto de servicio, las empresas de autobuses eran privadas, pero altamente subvencionadas y con un trato de favor en lo tocante al monopolio de las líneas, las rutas y los precios de carburante. Todo ello creaba un mapa de infraestructuras frente al tren, nacionalizado, y la red de carreteras, que daba directamente un trato de favor al coche privado, y que terminó de evidenciarse con la llegada de la democracia. Evidentemente, durante el franquismo se dejaron marcados los pasos para que el desarrollo del transporte fuera, cada vez más, un mapa del individualismo asociado al automóvil privado.

La estación de autobuses de Jaén está declarada como bien inmueble de interés histórico en el Catálogo de Patrimonio Andaluz (BOJA 2006), una significativa iniciativa política que ha permitido parar su destrucción y derribo, aunque no significa que no se siga cuestionado su uso, así como ambicionado el espacio que ocupa. Anteriormente a la estación de autobuses se usaba la Plaza del Deán Mazas como apeadero. En 1940 los arquitectos Laguna y López Rivera presentan un primer proyecto por encargo del Ayuntamiento de Jaén. Posteriormente se redacta un nuevo proyecto encargado por la compañía Auto-Estaciones a los arquitectos Sánchez Ballesta y de la Peña, que introducen un buen número de variaciones al proyecto de 1941. La dirección de obra la lleva el arquitecto Antonio Querejeta Rueda, tras el fallecimiento de Sánchez Ballesta. Es este arquitecto el que le introduce los gestos vernáculos e historicistas al edificio. Aunque no es lo que aquí tratamos es importante reconocer que el edificio tiene, en sintonía con el planteamiento de la época, un hotel añadido. Dado su mal estado, a causa principalmente de las goteras y su desfase tecnológico, estuvo al borde de la demolición en varias ocasiones, con el fin de construir una nueva estación y aparcamientos subterráneos, hasta que fue finalmente declarado como Bien de Interés Cultural y por obligación, restaurado en parte por el Ayuntamiento de Jaén y la Junta de Andalucía, si bien dicha restauración sólo supuso arreglos en la estación, no en el hotel anexo con el que conforma una unidad arquitectónica. A la vez que todo esto responde a una nueva mirada historicista y comprensivista de la arquitectura como parte de un patrimonio heredado, una idea de que el pasado vive apegado a lo que recibimos en forma de materiales, físicas y técnicas (González y Santofimia 2012; Shapiro 2011), una suerte de mecánica que hace del edificio proyectado desde el poder un asunto que conforma el "conservar" como verdadera forma de la identidad. Arquitectónicamente se trata de un proyecto con vocación claramente racionalista, como lo atestiguan las diversas propuestas que se suceden 
entre los años 1940 y 1945. Pretende formalizar las ideas del Movimiento Moderno extendidas por Europa con una arquitectura regional racionalista que facilitara la idea de funcionalidad. El edificio se compone de una gran sala de espera, que a su vez es el vestíbulo principal de la estación, sobre la que se levanta un hotel. Se cierra sobre sí mismo con un muro que crea el espacio para los andenes, y posee en la entrada una torre de reloj. La resolución de los remates en forma de curva, limpios de ornamento, es de una incontestable potencia formal muy característica de los planteamientos arquitectónicos del racionalismo. Destaca la estructura porticada del vestíbulo, prolongada hacia los andenes exteriores como una imponente marquesina volada (Casuso 1996 y 2001. Galera 2000; López 2000; Quesada y Casuso 2006).

En cualquier caso, la estación ha sido, y es, un punto neurálgico de la ciudad, que permite entender que Jaén capital es el centro regional, económico, administrativo y el polo de atracción social y cultural de un enorme territorio. De hecho, es curioso como mucha gente recuerda que la estación suponía, para muchos habitantes de los pueblos y ciudades de la provincia, venir a un Jaén relativamente moderno y caer en el centro de la ciudad, donde los edificios administrativos, institucionales, de educación o ocio, así como multitud de comercios de todo tipo, era un elemento clave de los planes e imaginarios con respecto a la vida en una sociedad construida en la idea de centros-periferias. Puede que al día de hoy la estación se encuentre habitada y usada por una corte de gente que vive en lo más vulnerable y débil económicamente de la sociedad (estudiantes y jóvenes, migrantes, mujeres trabajadoras y, sobre todo, por personas mayores) pero eso no quita, que entendamos el complejo proceso político y económico sobre el que se ha construido y hecho la genealogía de este espacio social. De hecho, todo esto es un producto en una sucesión de causasefecto de la decidida apuesta desde los años 30 por parte de los poderes del Estado por el transporte por carretera, frente a otros medios y maneras, lo que ha dado a los autobuses, altamente subvencionados, un potencial y centralidad determinante en la historia de la movilidad española (Martínez y Cobos 2006; Cuéllar 2001 y 2003). Pero a su vez y como ya decíamos, la estación ha sido un punto central también de las miradas locales, primero porque permite que la capital fuera el centro de una enorme comarca, donde se gestionan los servicios administrativos $y$ comerciales de un enorme entorno. $Y$, segundo, una manera discreta y asequible de viajar como parte de la emigración interior y, sobre todo, exterior.

\section{FLUIDOS EN LA FÍSICA DEL PODER}

En cualquier caso, la idea clave es seguro que tiene que ver con el fluir. En la estación la gente fluye, va de un sitio a otro. Durante el trabajo de campo, sentado en un banco, tenía la impresión que todo se parecía a un enorme éxodo, a una huida permanente, nada permanecía en el sitio. $Y$ nada parece contener ese constante ir y venir de sujetos, que como en un fluido se desparraman por todo el espacio sin que nada parezca contenerlos. Incluso las propias dinámicas arquitectónicas, planteadas para que se entre y salga por la única puerta principal, son rotas de manera permanente por los sujetos que utilizan la entrada y salida de los autobuses para salir más rápido. Fluidos en constante movimiento, sujetos a una dinámica que nos les permite ni ser fijados, ni establecerse en un único lugar, a la vez que desbordan toda la realidad espacial. La capacidad del fluir viene consiguiente determinada por la cantidad de personas que usan la estación. En el mundo de los ingenieros y arquitectos técnicos se estudia todo esto desde una razón que para los que acostumbramos a ver la realidad en pequeño resulta cuando menos sorpresivo. La adecuación entre las necesidades, el tamaño de la estación y los flujos, así como el volumen de población de la ciudad, el tipo de servicio que se ofrece y la capacidad material son variables que terminan por configurar esa doble realidad de ser parte de la ingeniería civil y, a la vez, una administración empresarial (Millán 2001: 21-23), que parece ser a lo que reduce una estación el mundo técnico, un espacio arquitectónico lleno de dársenas y con una funcionalidad de carácter empresarial.

Pero en el fluir de la gente, la dársena no es sólo un espacio en el que el autobús se detiene para dejar o recoger viajeros, es un lugar de mirada, un espacio que se observa y que sirve, consiguientemente, de frontera entre dos principios, el del autobús y el de ese espacio natural que es la estación. La dársena ya no es sólo el lugar del autobús, sino el lugar donde el viajero deja de fluir, deja de ser un hecho y empieza a ser un sustantivo, el espacio de transformación de ser un cuerpo activo a estar en un cuerpo pasivo. Se pasa de ciudadano-consumo a cuerpo viajero. Digamos que la dársena no es tanto un espacio arquitectónico 
sino una frontera, una raya que delimita hasta dónde alguien es un sujeto y qué ocurre luego siendo Otro; un transmutador que permite situar tanto las mecánicas de la subjetividad, como las prácticas subjetivas. El fluir de la estación tiene, consecuentemente, algo de automático y es sin duda un lugar que muestra con una cierta certeza las enormes capacidades de naturalizar y asumir la urbanidad, la disciplina y el control que se ejercen constantemente sobre el corpus social. Entrar y comprar el billete de manera diligente, subir, bajar o meter las maletas o esperar de manera ordenada remarcan las dos ideas de lo que entendemos es vivir en una sociedad con un proyecto civilizatorio: guardar el turno según el criterio temporal de llegada, no forzando a los demás y pagar el autobús, en cuanto servicio, entendiendo que cada sujeto es una individualidad. Es decir, la estación de autobuses remarca una idea permanente de individualidad en el eje uso-consumo y en el enorme disciplinamiento que se efectúan en el mundo social. Y este hecho, o este conjunto de hechos, más bien naturalizados y asumidos, se hacen en tanto que se está fluyendo.

No se fluye, en cuanto que los sujetos van de un lado a otro, sino que es en una dinámica del movimiento, en una serie de itinerarios marcados y con unas prácticas asociadas. En la estación de autobuses no se establece nada que no esté dentro de una posible finalidad dada, contenerse o des-contenerse de un autobús. Este principio rector no es tanto una realidad que se establece desde algún lugar, idea o concepto con respecto a la funcionalidad de la estación de autobuses: qué otra cosa podría ser una estación sino lo que parece, una instalación funcional dedicada a la gestión de los viajes. Sin embargo, no es sólo eso, o por lo menos eso es sólo lo que parece, pues el aparato disciplinar de cómo se hace y cómo se vive esa funcionalidad y gestión determina que estamos ante un espacio muy significativo de la vida social de la ciudad que la mantiene. La significación de la estación no sólo es que los sujetos que la usan la vean como una aplicación y un símbolo, sino también el profundo sentido histórico y contextual que todo ello supone: no en vano resume la historia reciente de la ciudad, la forma en cómo desde hace casi un siglo se establece la cotidianidad entre lo que los sujetos sociales consideran público o privado e incluso, lo que muchos determinan como privativo. La estación es también el resultado de unas políticas que han hecho de la tecnología su centro, una tecnología que hace del automóvil, el transporte, algo empresarial, individual y normativo. Pero estas tecnologías son, en lo más significativo, formas mecanizadas en las vidas de la cotidianidad, donde los sujetos se convierten en cierta medida en mecanismos corporales, disciplinados, controlados y normativizados, en un fluir, eso sí, como partículas en una reconocida y constante microfísica.

Quizás el hombre moderno, que se soñó en la República de Weimar (Benjamin 2009: 85-137), fuera aquel que aunaba el concepto de movilidad al de espacio, moverse en cierta medida requiere de la idea de que el espacio está lo suficientemente culturizado como para poder desplazarse por él. Pero el sujeto contemporáneo es más complejo y está cristalizado en muchos otros elementos que le fijan a realidades por las que es difícil moverse. Esta idea de que los sujetos se desplazan para ir de aquí para allá, no es más que una metáfora del ir para aquí en un contexto mercantil y en un espacio privativo, donde la idea del movimiento les fija a un sedentarismo activo, el llamado sedentarismo nómada, que analíticamente es rotacional, individual, mecánico y masivo (Bericat 1994: 115-120), y que aquí en la estación de autobuses es tan obvio, al observar la imposibilidad de fijar a los sujetos a un espacio con el que sólo se pueden relacionar en la medida que se desplazan para ir o volver siempre a un espacio dado de antemano. Partículas en constante movimiento rotacional que sedentarizan sus realidades, flujos y contenciones, controles y particularidades, todo parece el guión de una enorme obra de teatro donde los sujetos se convierten en elementos individuales, sin más posibilidad de vivir que ser parte de un entramado mayor de dispositivos de contención, de dinámicas de fluidos y de gestiones energéticas. En el fondo la estación es una presa que con forma de dique contiene, almacena y gestiona partículas líquidas; pero seguramente es también la metáfora de una sociedad donde el movimiento social, de clase, de género, de nacionalidad, es tan verdadero como poco probable. La estación de autobuses en su empeño de convertirse en el nexo comunicativo, no deja de ser un espacio donde la movilidad social muestra la cara de la vida social, la movilidad como un imposible. Los sujetos, acaso, rotan por el espacio dando a la movilidad un contenido semántico que supone, en la medida que la regla es poder ser libre, poder ir a todos los sitios a los que obviamente nunca se irá. Como metáfora de lo social la estación de autobuses muestra que es posible ir a casi cualquier sitio, aunque 
una vez en el interior de la estación las personas sólo pueden ir a un sitio, en el mejor de los casos al que les corresponde, en el peor a la incertidumbre, ese lugar donde sólo se puede ser una partícula, un radical libre en búsqueda de oxígeno.

\section{UN RELATO SIN FUTURO}

La imagen de la estación de autobuses de Jaén, aunque podría decirse de otras, es un edificio viejo que no cumple con rigor con la funcionalidad con que fue ideado. Aunque tras su aparataje técnico se esconde un fluir que da forma tekno-lógica, en qué se ha de operar sobre lo social y dónde se subrayan todas aquellas ideas asociadas a la empresa privada, la individualización y la mercantilización. De alguna manera, la imagen de que se trata de un viejo edificio es más bien un efecto óptico que otra cosa, digamos que es una construcción poco cuidada, nada conservada y con un planteamiento arquitectónico muy alejado de los actuales edificios asépticos de cristal y aluminio. Sin embargo, la estación de autobuses es en muchos sentidos un proyecto que contextualiza muy bien el concepto de modernidad con el que se construyó la España victoriosa de la posguerra, donde el pacto de crear una sociedad civil pasaba por dos puntos: dejar la economía en manos de las empresas privadas y dos, dar la oportunidad a que la sociedad cada vez fuera más individual, fluida y aséptica. Consecuentemente, la estación de autobuses creaba una imagen de victoria, de fuerza, de atención a los nuevos principios del nacionalcatolicismo que servían de base al franquismo, con su centralismo y juego de centros periferias, de niveles de desarrollo y de subordinación. Pero además, dejaba en manos de la empresa privada su construcción y explotación, la gestión de un servicio social que sería clave para el modelo de vertebración social y que permitiría, a la postre, establecer las bases que darían la enorme reconfiguración del mapa social, demográfico y político que se estaba gestando, permitiendo pero a la vez controlando y disciplinando, los movimientos de las personas por el territorio nacional.

La estación de autobuses cumplió, en aquellos primeros momentos de su historia, además, con otros dos elementos que resaltan la modernidad y con los que fue planteada: por un lado, la gran capacidad de normalización de todo lo que tenía que ver con el transporte, permitiendo un control de las empresas, la carga y los viajeros. En última instancia, no puede darse una gestión de la sociedad sino es, primeramente, controlando sus flujos y las formas en cómo se asume la idea de movilidad. La estación de autobuses, con su enorme capacidad de objetivar a los viajeros y sus enseres, permite un control y disciplinamiento que es clave para la idea de modernidad que se da en las zonas periféricas del Estado español, primero franquista, y luego en la democracia. Desde la persecución del estraperlo, esa forma de economía sumergida que permitió que las clases sociales beneficiarias de la victoria franquista se saltaran los férreos controles de bienes que había establecido el Estado, hasta el control y gestión de los flujos migratorios de los años 50 del siglo XX, pasando por las más que actuales implementaciones de la capitalización y centralidad de la política provincial y local, lo que ha sido causa efecto de que Jaén pueda tener una Universidad propia o el control, muy sutil, de la emigración africana, articulando la movilidad en esta zona que vive de la doble explotación de la tierra y quién la trabaja (un ejemplo paralelo para Castilla-La Mancha está en Fernández Santamaría 2000). Y por otro, la puesta en marcha, la exaltación y desarrollo del coche particular, el gran ganador, símbolo y finalidad de las políticas y economías del capitalismo occidental (Anta 2015; Miller 2020). En cierta medida si hay algo de permanente decadencia en la estación de autobuses, es la falta de una cierta mirada de actualidad, que se expresa en la idea de que es un espacio viejo y usado, debido a que la estación de autobuses se ha quedado como un espacio donde se representa el drama de las clases sociales. Un lugar donde trabaja la idea de que se usan los servicios públicos en función del lugar que se ocupa socialmente. Los mundos poscapitalistas, seguramente, ya no funcionan en sintonía con lo que proponen, ofrecer un servicio universal, sino en la medida que prometen elementos que muestran la vocación de ser parte de una distinción ideológica y económica, en última instancia, de clase.

Pero la estación de autobuses no puede resistirse a una mirada más pequeña, y esto revela que la estación es utilizada por un número creciente de personas en los márgenes sociales, económicos, de género, de edad o de carácter étnico. Lo que no puede ser sólo un efecto de las crisis económicas, sino más bien el lugar en que se plantea una modernidad universal adaptando a las personas a sus posibles velocidades. No podemos pensar de otra manera cuando leemos, en otras etnografías sobre las estaciones de autobuses 
(Meir-Dviri y Raz 1995; Callahan 2013), que todo esto no es otra cosa que un espacio donde determinados grupos sociales marginados, en los estudios clásicos pedigüeños y limosneros, ocupan su tiempo y energías en ser visualizados, protegidos y adscritos. De manera más local podemos ver que aquí la apuesta por el transporte por carretera y por la gestión empresarial privativa del transporte, dieron con una imagen concreta de quién usa qué medio de transporte y para qué. En cierta medida la estación de autobuses no es un elemento que se pueda estudiar si no es en relación a su cambiante y fluida historia, así como el lugar que, al configurar las políticas de la movilidad, estructurando la ciudad de una manera precisa y concreta (Julià 2006); donde se termina por configurar una imagen de la vida urbana centrada en que todo es, en definitiva, una parte de un itinerario donde sólo se transita (Delgado 2007). Ser ciudadano, ser parte de la ciudad, es más un estar en alguna posición relativa que una admisión de la identidad, acaso sólo cuando se consume se tiene un momento de subjetividad y puede ser, el momento en que se forma parte de una comunidad. Quizás el único momento exacto en que todo tiene sentido, conformando una unidad con el resto del cuerpo social, es el poco tiempo en que se está parado esperando la llegada del autobús o a comprar el billete. Un breve momento de contacto con lo social donde se podría ver toda la maquinaria en funcionamiento, pero dura tan poco, es tan improbable, está tan cerrada por el consiguiente acto de consumo que es una experiencia aparentemente intrascendente y no acumulativa. Aún así abre un hilo de esperanza, un intersticio minúsculo sobre el asfalto, estipula la posibilidad de otra sociedad diferente.

Sin embargo, como proyecto social de modernidad la estación de autobuses es la imagen de un fracaso: lo público se ha convertido sólo en un espacio donde opera la empresa privada subvencionada. De hecho, en la estación de autobuses, como un lugar privilegiado del capitalismo, de sus políticas y economías, se puede ver con claridad quién es la bestia, qué come y qué mano le alimenta. Una vez que se mercantilizan los servicios, espacios e instituciones sociales, el Estado de Bienestar está en otros parámetros que los que establece una serie de derechos para los ciudadanos (Andrade et al. 2000; Soriano et al. 2011). Y se torna todo en una política encaminada a satisfacer necesidades que se cuantifican en apuntes contables que tienen que dar, si o si, beneficios. Pero quizás detrás de este espacio hay otras verdades sociales, culturales y políticas. Seguramente es importante entenderlo como un lugar donde se gestionan los riesgos, ahora ya de un orden pacífico y tecnológico, y donde lo social se disciplina en una red de acuerdos normativos en torno a elementos complejos y vulnerables (Beck 2000: 78-82). Mover cuerpos tiene algo de riesgoso, de un riesgo que la estación de autobuses gestiona como si de una moderna tecnología se tratará, calculando los movimientos, los tiempos, los espacios en una eficacia que quizás sólo sea eso: un control de los riesgos, de la vulnerabilidad de los cuerpos, de las prácticas e inseguridades industriales, mecánicas y de la incertidumbre institucional y empresarial.

Sin duda, la estación de autobuses es también el lugar de la gestión de múltiples seguros, un invento muy moderno, que hace un cálculo de la fatalidad como algo inevitable, seguro de automóvil, de transporte, de protección civil y de uso y disfrute del espacio público que reconoce, por medio de estos mecanismos, la idea de que son privados y privativos, gestionados y controlados. La estación de autobuses se convierte en un espacio que calcula el riesgo y convierte a los viajeros y usuarios en un número probabilístico, un "riesgo" que estadísticamente es predecible. Al reducir a los sujetos a su propia corporalidad de la estadística, a la variable que le determina como objeto de la mirada cientificista, se suprime el "comportamiento social" y se reduce todo a una individualización del daño. El seguro, que la estación de autobuses gestiona, no acaba con el riesgo, simplemente, lo canaliza hacia el individuo; eso sí ya convertido en cuerpo de y para el consumo y que carece de otra posibilidad que de ser un hecho comercial. A la vez que la estación de autobuses una vez más da esa apariencia de público cuando es sólo privada, un espacio políticamente intermedio entre las ideas del socialismo protector, previsor y garantista y el neoliberalismo empresarial encargado de generar una constante de riesgos que son la nueva y más efectiva manera de crear beneficios.

Por otro lado, la estación de autobuses de Jaén nos plantea una posibilidad de entender este tipo de espacios transtradicionales (Giddens 1997) como una dinámica social compleja, contradictoria y creativa. Durante décadas corrió la idea de que los servicios de la estación eran un lugar donde ancianos pederastas y hombres homosexuales de mediana edad venían en búsqueda de jóvenes a los que masturbar (la idea tiene más a más, un correlato trágico en la denuncia de una 
mujer por violación en este espacio en febrero del 2014). Durante tanto tiempo escuché este mito urbano, que se extiende por todas las estaciones de transporte público del mundo capitalista (Mileski y Black 1972; Sundholm 1973), que llegué a creer que era cuando menos una enorme exageración. Sin embargo, qué puede ser una estación de autobuses sino el lugar exacto donde todos los viajes son posibles, una frontera en donde viven las normas de una sociedad y los pasadizos que llevan a otros mundos, un lugar lleno de resquicios, y donde la sociedad propone un encuentro de múltiples elementos. Si la estación representa el lugar del encuentro, de la despedida, de las idas y venidas, sus servicios públicos no pueden ser otro sitio que el lugar privativo de lo que la experiencia viajar propone, el encuentro con un desconocido, la posibilidad de que la vida en los límites de la sociedad normalizadora y civilizadora den lugar a espacios de anonimato, de gozo y de experimentación. La modernidad tiene estas cosas, propone en todas las direcciones: mientras construye formas de disciplina que exige controles en sus dársenas, a la vez, ofrece un servicio público que deja la puerta abierta a los encuentros fortuitos, a las mitologías sexuales liberadoras y vigilantes. Quizás, por esto mismo el servicio de la estación fue controlado, limpiado y vigilado por un inválido de guerra-obviamente del bando vencido de la Guerra Civil-que desde la humillación del trabajo en un lugar apartado y sucio recibía una moneda del Estado, para que sirviera de chivato, y otra del homosexual de turno, para que mirara para otro lado. En definitiva, esta estación del Sur, de todos los sures, es un experimento de la movilidad contemporánea poscapitalista, pero ante todo de la gestión de las corporalidades en un lugar fronterizo y extremo.

\section{BIBLIOGRAFÍA CITADA}

Andrade Fernández, Elena, Constantino Arce Fernández y María José Ferraces Otero. 2000. «Valoración del autobus como medio de transporte público urbano un estudio de las diferencias entre segmentos de la población». Revista de Psicología Social 15(3): 335-342.

Anta Félez, José-Luis. 2013. "Una etnografía del avión. Cuerpossujetos a la disciplina del consumo viajero». AIBR. Revista de Antropología Iberoamericana 8(3): 323-344.

Anta Félez, José-Luis. 2015. «El automóvil: genealogía de un objeto de poder». Methaodos. revista de ciencias sociales 3(1): 93-106

Augé, Marc. 1993. Los no lugares: Espacios de anonimato. Una antropología de la sobremodernidad. Barcelona: Gedisa.
Beck, Ulrich. 2000. La sociedad del riesgo global. Madrid: Siglo XXI.

Benjamin, Walter. 2009. La dialéctica en suspenso. Santiago de Chile: LOM.

Bericat, Eduardo. 1994. Sociología de la movilidad espacial. El sedentarismo nómada. Madrid: Siglo XXI-CIS.

BOJA. 2006. «Resolución de 21 de febrero de 2006, de la Dirección General de Bienes Culturales, por la que se resuelve inscribir colectivamente, con carácter genérico, en el Catálogo General del Patrimonio Histórico Andaluz, nueve bienes inmuebles del Movimiento Moderno de la provincia de Jaén». Boletín Oficial de la Junta de Andalucía 51: 53-64. Sevilla: Junta de Andalucía.

Callahan, Laura. 2013. «Requests for Money on Public Transportation: Saving Face for Speaker and Hearers». Prisma Social 10: 361-393.

Castilho, Denis. 2012. «Estado e rede de transportes em GoiásBrasil (1889-1950)». Scripta Nova. Revista Electrónica de Geografía y Ciencias Sociales XVI, 418 (67).

Casuso Quesada, Rafael Antonio. 1996. «La estación de Autobuses de Jaén: una importante aportación a la arquitectura española de posguerra». Senda de los Huertos 41: 59-69.

Casuso Quesada, Rafael Antonio. 2001. «Propuestas para una revisión crítica de la arquitectura del siglo XX en Jaén». En Fernández, José (ed.), Homenaje a Luis Coronas: 87-177. Jaén: Universidad de Jaén.

Chambers, lain. 1986. Popular Culture. the Metropolitan Experience. Londres-Nueva York: Routledge.

Crespo Guerrero, José M. y Egidio Moya García. 2012. «Los transportes colectivos como dinamizadores culturales». En Miramontes Carballada, Angel, Royé, Dominic y Villa Vázquez, José Ignacio (Coords.), Las ciudades y el transporte urbano. Reflexiones en tiempos de crisis: 307-319. Santiago de Compostela: Meubook.

Cuéllar Villar, Domingo. 2001. «La Mecanización del Transporte por Carretera: Redes y Empresas en el Sureste Andaluz (19001950)». VII Congreso de la Asociación de Historia. Zaragoza.

Cuéllar Villar, Domingo. 2003. Los Transportes en el Sureste Andaluz (1850-1950). Economía, Empresas y territorio. Madrid: Junta de Andalucía, Cajamar, Fundación de los Ferrocarriles Españoles.

D’Aubeterre, Luis. 2012. "Sobre la temporalidad discursiva y el sentido común venezolano en un pullman: Un ensayo de etnografía hermenéutica». Espacio Abierto. Cuaderno Venezolano de Sociología 21(3): 480-501.

Dalmaso, Sandra A. R. y Maria C. Coutinho. 2010. «Tecnologia e trabalho: Sentidos produzidos no cotidiano do transporte coletivo». Arquivos Brasileiros de Psicologia 62(3): 93-104.

Daniels, Peter W. y A. M. Warnes. 1983. Movimiento en ciudades: transporte y tráfico urbanos. Madrid: Instituto de Estudios de Administración Local.

Delgado, Manuel. 2007. Sociedades movedizas. Pasos hacia una antropología de las calles. Barcelona: Anagrama.

Fernández Durán, Ramón. 1980. Transporte, Espacio y Capital. Madrid: Nuestra Cultura. 
Fernández Santamaría, Francisco. 2000. Transporte publico de viajeros y accesibilidad en la provincia de Albacete. Cuenca: Universidad de Castilla La Mancha.

Frax Rosales, Esperanza y Santos Madrazo. 2001. « «El transporte por carretera, siglo XVII-XX». TST: Transportes, Servicios y telecomunicaciones 1: 31-53.

Galera, Pedro A. 2000. "Jaén, la ciudad del siglo XX». Senda de los Huertos 57-60, I: 21-31.

Giddens, Anthony. 1997. "Vivir en una sociedad postradicional". En Beck, Ulrich, Anthony Giddens y Scott Lash (eds). Modernidad reflexiva. Política, tradición y estética en el orden social moderno: 75-136. Madrid: Alianza.

Giucci, Guillermo y Tomás Errázuriz. 2018. El viaje colectivo. La cultura del tranvía y del ómnibus en América del Sur. Talca: Campus Creativo.

Goffman, Erving. 1991. Los momentos y sus hombres. Barcelona: Paidós.

González Martínez, Plácido y Marta Santofimia Albiñana (Coords.). 2012). Cien años de arquitectura en Andalucía: el Registro Andaluz de Arquitectura Contemporánea, 19002000. Sevilla: Consejería de Cultura.

Güller, Michael y Mathis Güller. 2002. Del aeropuerto a la ciudad-aeropuerto. Barcelona: Gustavo Gili.

Gutiérrez, Andrea. 2010. «Movilidad, transporte y acceso: una renovación aplicada al ordenamiento territorial». Scripta Nova. Revista Electrónica de Geografía y Ciencias Sociales XIV, 331: 86 .

Jacobs, Jane. 2011. Muerte y vida de las grandes ciudades. Barcelona: Capitan Swing.

Herce Vallejo, Manuel. 2009. Sobre la movilidad en la ciudad: propuestas para recuperar un derecho ciudadano. Barcelona: Reverte.

Herce Vallejo, Manuel. 2010. «Las infraestructuras como instrumento de orden territorial y equidad social». Cuestiones urbanas 1: 57-77.

Herce Vallejo, Manuel y Joan Miró Farrerons. 2002. El soporte infraestructural de la ciudad. Barcelona: Universitat Politècnica de Catalunya.

Horta, Gerard. 2019. "From Peripatetic Anthropology to the Ethnography of Roads and Motorisation in Africa ". Cadernos de Estudos Africanos 37: 213-233.

Lefebvre, Henri. 1976. Espacio y política. Barcelona: Península.

López Arandia, María Teresa. 2000. «Una mirada a la arquitectura giennense en el siglo XX». Senda de los Huertos 57-60, II: 369-382.

Martínez, Tomás y Francisco de los Cobos. 2006. «Competencia y coordinación ferrocarril-carretera. Una visión desde la década de los treinta: Francisco Jiménez Ontiveros». IV Congreso Historia Ferroviaria. Málaga.

Meir-Dviri, Mina y Aviad E. Raz. 1995. «Rituals of Exchange in the Social World of Israeli Beggars: an Exploratory Study». Symbolic Interaction 18(2): 99-119.
Miller, Daniel (ed.). 2020. Car cultures. Nueva York. Routledge.

Mileski, Maureen y Donald J. Black. 1972. «The Social Organization of Homosexuality». Urban Life and Culture 1(2): 187-202.

Millán Rincón, Juan. 2001. Estaciones y apeaderos de autobuses en Andalucía. Sevilla: Consejería de Obras Públicas y Transportes.

Millán Rincón, Juan. 2006. "La Red de estaciones de autobuses de Andalucía». Revista Carreteras 146: 31-41.

Julià Sort, Jordi. 2006. Redes metropolitanas. Barcelona: Gustavo Gili.

Montes Cañete, Macarena. 1999. "La historia del autocar durante la década de los años 50». Viajero 45: 26-30.

Natal, Jorge. 2003. Transportes, território e desenvolvimento econômico. Rio de Janeiro: Papel Virtual.

Olalla, Vicente. 1977. «Estaciones de autobuses». Informes de la Construcción 29, 289: 3-49.

Potthast, Joerg. 2012. «Terminal». En Marquardt, Nadine y Verena Schreiber (ed.), Ortsregister: Ein Glossar zu Räumen der Gegenwart: 273-279. Bielefeld: Transcript.

Quesada García, Santiago y Rafael Antonio Casuso Quesada. 2006. La Estación de Autobuses de Jaén. una Topografía de su Arquitectura. Sevilla: Consejería de Cultura de la Junta de Andalucía.

Seijo Marcos, Begoña. 2006. «Del tren al autobús: la modernización del transporte público en España en la década del desarrollismo». IV Congreso Historia Ferroviaria. Málaga.

Seguí, Joana María y María Rosa Martínez. 2004. Geografía de los Transportes. Palma de Mallorca: Universidad de les Illes Balears.

Shapiro, Kelli K. 2011. Saving Suburban Sites and Rescuing Roadside Relics: the Historic Preservation of the Recent Past Through Adaptive Reuse. Providence, Rhode Island: Brown University.

Soriano García, Carol, Paula Cembrero Coca, Nadia Campos Soriano, José S. Solaz Sanahuja, Elisa Signes i Pérez y José Joaquín Tarazaga. 2011. «Percepción de la imagen del transporte de viajeros en autobús potencialidades y acciones de mejora». Revista de Biomecánica 55: 15-18.

Sundholm, Charles A. 1973. «The Pornographic Arcade: Ethnographic Notes on Moral Men in Immoral Places». Urban Life and Culture 2(1): 85-104.

Taplin, Dana H., Suzanne Scheld y Setha M. Low. 2002. «Rapid Ethnographic Assessment in Urban Parks: a Case Study of Independence National Historical Park». Human Organization 61(1): 80-93.

Urry, John. 1999. Sociology beyond societies: Mobilities for the Twenty-First Century. Londres: Routledge.

Urry, John. 2007. Mobilities. Cambridge: Polity.

Velasco, Honorio y Ángel Díaz de Rada. 2009. La lógica de la investigación etnográfica. Madrid: Trotta.

Zárate Martín, Manuel Antonio. 1997. Ciudad, Transporte y Territorio. Madrid: UNED. 